\title{
Bicycling Suppresses Abnormal Beta Synchrony in the Parkinsonian Basal Ganglia
}

\author{
Lena Storzer, PhD, ${ }^{1}$ Markus Butz, PhD, ${ }^{1}$ Jan Hirschmann, PhD, ${ }^{1,2}$ \\ Omid Abbasi, PhD, ${ }^{1,3}$ Maciej Gratkowski, PhD, ${ }^{4}$ Dietmar Saupe, PhD, ${ }^{4}$ \\ Jan Vesper, MD, $\mathrm{PhD}^{5}$ Sarang S. Dalal, $\mathrm{PhD}^{6,7 \star}$ and Alfons Schnitzler, MD, $\mathrm{PhD}^{1,8 *}$
}

Objective: Freezing of gait is a poorly understood symptom of Parkinson disease, and can severely disrupt the locomotion of affected patients. However, bicycling ability remains surprisingly unaffected in most patients suffering from freezing, suggesting functional differences in the motor network. The purpose of this study was to characterize and contrast the oscillatory dynamics underlying bicycling and walking in the basal ganglia.

Methods: We present the first local field potential recordings directly comparing bicycling and walking in Parkinson disease patients with electrodes implanted in the subthalamic nuclei for deep brain stimulation. Low $(13-22 \mathrm{~Hz})$ and high $(23-35 \mathrm{~Hz})$ beta power changes were analyzed in 22 subthalamic nuclei from 13 Parkinson disease patients ( $57.5 \pm 5.9$ years old, 4 female). The study group consisted of 5 patients with and 8 patients without freezing of gait. Results: In patients without freezing of gait, both bicycling and walking led to a suppression of subthalamic beta power $(13-35 \mathrm{~Hz})$, and this suppression was stronger for bicycling. Freezers showed a similar pattern in general. Superimposed on this pattern, however, we observed a movement-induced, narrowband power increase around $18 \mathrm{~Hz}$, which was evident even in the absence of freezing.

Interpretation: These results indicate that bicycling facilitates overall suppression of beta power. Furthermore, movement leads to exaggerated synchronization in the low beta band specifically within the basal ganglia of patients susceptible to freezing. Abnormal $\sim 18 \mathrm{~Hz}$ oscillations are implicated in the pathophysiology of freezing of gait, and suppressing them may form a key strategy in developing potential therapies.

$T_{\text {hestis }}^{\text {he }}$ he basal ganglia are a key player in the locomotor network interacting with cortical motor, cerebellar, and brainstem structures and consequently, gait impairments are a main problem in Parkinson disease. ${ }^{1}$ It is well known that oscillatory activity in the corticobasal ganglia loop, especially in the beta band $(13-35 \mathrm{~Hz})$, is altered in Parkinson disease, ${ }^{2}$ and it has been proposed that beta oscillations promote the maintenance of the motor state at the expense of voluntary movement. ${ }^{3}$
Walking abilities are especially affected in patients suffering from freezing of gait (FOG), a common phenomenon in advanced Parkinson disease. FOG is defined by the episodic inability of forward progression despite the intention to walk. ${ }^{4}$ In addition, these patients also show an abnormal spatiotemporal gait pattern in between freezing episodes including smaller steps at higher cadence. ${ }^{5,6}$ Strikingly, most Parkinson disease patients, with and without FOG, were found to maintain the

*These authors contributed equally to this work.

Address correspondence to Dr Butz, Institute of Clinical Neuroscience and Medical Psychology, Medical Faculty, Heinrich Heine University Düsseldorf, Universitätsstraße 1, 40225 Düsseldorf, Germany. E-mail: Markus.Butz@hhu.de

From the ${ }^{1}$ Institute of Clinical Neuroscience and Medical Psychology, Medical Faculty, Heinrich Heine University Düsseldorf, Düsseldorf, Germany; ${ }^{2}$ Donders Institute for Brain, Cognition, and Behavior, Radboud University, Nijmegen, the Netherlands; ${ }^{3}$ Department of Medical Engineering, RuhrUniversity Bochum, Bochum, Germany; ${ }^{4}$ Department of Computer and Information Science, University of Konstanz, Konstanz, Germany; ${ }^{5}$ Department of Functional Neurosurgery and Stereotaxy, Medical Faculty, University Hospital Düsseldorf, Düsseldorf, Germany; ${ }^{6}$ Center of Functionally Integrative Neuroscience, Aarhus University, Aarhus, Denmark; ${ }^{7}$ Zukunftskolleg and Department of Psychology, University of Konstanz, Konstanz, Germany; and ${ }^{8}$ Center for Movement Disorders and Neuromodulation, University Hospital Düsseldorf, Düsseldorf, Germany 
ability to bicycle. ${ }^{7,8}$ This is puzzling with respect to our understanding of the human motor system and strongly suggests that, despite the similarity in their rhythm and involved muscle groups, ${ }^{9}$ bicycling and walking may involve cerebral structures including the basal ganglia in different ways.

There is evidence linking FOG to the excessive synchronization in the basal ganglia caused by reduced levels of dopamine in combination with temporarily increased motor, cognitive, or limbic load. ${ }^{10,11}$ In line with this, abnormally synchronized basal ganglia beta band oscillations have been found in patients with FOG both during rest $^{12}$ and during walking. ${ }^{13}$ Whereas nonfreezers displayed the characteristic power reduction across the entire beta band in the subthalamic nucleus (STN) during walking, oscillatory activity specifically in the low beta band $(12-22 \mathrm{~Hz})$ was increased in freezers. ${ }^{13}$ These findings are consistent with the assumed antikinetic nature of oscillatory beta activity. However, the question remains how exactly this difference in movement-related beta power changes is related to FOG. Is this difference specific to gait or is it rather a general signature of altered basal ganglia activity that distinguishes freezers from nonfreezers?

As no study to date has examined basal ganglia activity during bicycling, or how it may differ from walking, potential mechanisms concerning how bicycling abilities are preserved in Parkinson disease have remained speculative. It is still not entirely clear whether the basal ganglia are critically involved in the control of bicycling at all, and if so, how the underlying oscillatory activity may be modulated. Recently, our group demonstrated that healthy adults exhibit stronger sensorimotor high beta power $(23-35 \mathrm{~Hz})$ suppression in bicycling than in walking. ${ }^{14}$ We hypothesized that bicycling attenuates pathological oscillations also within the basal ganglia of parkinsonian patients as compared to walking. To test this hypothesis, we recruited Parkinson patients with and without FOG who had undergone implantation of electrodes for deep brain stimulation (DBS) therapy. This approach provides the rare opportunity to record local field potentials (LFPs) directly from the STN while initiating, sustaining, and terminating pedaling on a stationary bicycle or walking.

\section{Patients and Methods}

\section{Patients and Surgery}

Seventeen Parkinson disease patients clinically selected for bilateral DBS therapy participated in this study. One patient terminated the recording after the bicycling task due to fatigue and did not perform the walking task. One patient was inadvertently recorded in the medication $\mathrm{ON}$ state and was thus excluded from the analysis. Data of 2 patients had to be excluded because of severe movement artifact contamination of all STN channels. Thus, data are presented from the remaining 13 patients (age $=57.5 \pm 5.9$ years, 4 female, Table). The study was approved by the local ethics committee of the Medical Faculty of the Heinrich Heine University Düsseldorf (study number 4294) in accordance with the Declaration of Helsinki. All patients gave their prior written informed consent.

Motor state and history of FOG were assessed preoperatively with part III of the Unified Parkinson's Disease Rating Scale and the Freezing of Gait Questionnaire (FOG-Q). ${ }^{15}$ Cognitive and emotional states were assessed with the Mattis Dementia Rating Scale, the Frontal Assessment Battery, and the Becks Depression Inventory. Five patients were classified as freezers because of a score $>1$ regarding the FOG-Q question \#3: "Do you feel that your feet get glued to the floor while walking, turning or when trying to initiate walking?" Additionally, we observed freezing episodes in 2 of these 5 patients during the experiment. These episodes were excluded from the analysis.

Surgery was performed at the Department of Stereotaxy and Functional Neurosurgery of the University Hospital Düsseldorf as described earlier. ${ }^{16}$ Six patients (\#3, 5-9) were bilaterally implanted with a rechargeable Vercise DBS system (model 2201, Boston Scientific, Marlborough, MA). Patient \#11 was implanted with a Boston Scientific Vercise PC stimulator and had segmented directional leads with 8 contacts arranged in 2 ring contacts and two 3-segment contacts (1-3-3-1, model 2202-DL). The remaining 6 patients (\#12-17) were implanted with the Infinity DBS system (model 6170, St. Jude Medical, Saint Paul, MN), which is also equipped with segmented leads (1-3-3-1).

\section{Experimental Design and Procedure}

Recordings took place 1 to 5 days after electrode implantation using externalized leads. Patients were off oral dopaminergic medication for $\geq 12$ hours and off subcutaneous apomorphine administration for $\geq 2$ hours.

Patients engaged in bicycling on a stationary bicycle simulator, over-ground walking, and the respective baseline conditions, that is, sitting and standing (Fig 1). Patients started with a 120 -second baseline rest period. This was followed by repeatedly alternating 10 seconds of rest and 10 seconds of movement. This way, 30 episodes of acoustically cued movement initiation and termination were captured. At last, patients engaged in 120 seconds of continuous movement. Patients started either in the bicycling or in the walking condition with the order of conditions being pseudorandomized and counterbalanced across patients. For a more detailed description of the paradigm and the bicycle simulator, see Gratkowski et al. ${ }^{17}$ Patients were instructed to bicycle and to walk at their own comfortable cadence, as physical fitness was heterogeneous in the patients under study. Cadence was assessed in bicycling and walking as revolutions per minute (rpm) and strides per minute (spm), respectively. Patients were asked to keep cadence constant across conditions. 


\begin{tabular}{|c|c|c|c|c|c|c|c|c|c|}
\hline Patient & Age/Sex & $\begin{array}{l}\text { Disease } \\
\text { Duration, } \\
\text { yr }\end{array}$ & Subtype & $\begin{array}{l}\text { UPDRS III } \\
\text { OFF Scores }\end{array}$ & $\begin{array}{l}\text { L-dopa } \\
\text { Equivalent } \\
\text { Dose, } \\
\text { mg/Day }\end{array}$ & $\begin{array}{l}\text { Selected } \\
\text { Contacts }\end{array}$ & $\begin{array}{l}\text { MNI } \\
\text { Coordinates } \\
\text { of Selected } \\
\text { Contact, X, Y, Z }\end{array}$ & $p^{\mathrm{a}}$ & $\begin{array}{l}\text { Individual } \\
\text { Beta } \\
\text { Frequency, } \\
\mathrm{Hz}\end{array}$ \\
\hline \multirow[t]{2}{*}{3} & $61 / \mathrm{M}$ & 7 & Hypokinetic-rigid & 15 & 980 & L 5 vs 6 & $-12.5,-14.5,3$ & $0 \%$ & 15 \\
\hline & & & & & & R 4 vs 5 & $15.5,-14,-2$ & $0 \%$ & 16 \\
\hline \multirow[t]{2}{*}{5} & $46 / \mathrm{M}$ & 8 & Hypokinetic-rigid & 20 & 930 & L 2 vs 3 & Not available & & 34 \\
\hline & & & & & & R 2 vs 3 & & & 16 \\
\hline \multirow[t]{2}{*}{$6^{\mathrm{b}}$} & $62 / \mathrm{F}$ & 14 & Hypokinetic-rigid & 15 & 1,080 & L 3 vs 4 & $-9.5,-15.5,-7$ & $38 \%$ & 27 \\
\hline & & & & & & R 3 vs 4 & $12,-14.5,-7.5$ & $25 \%$ & 20 \\
\hline 7 & $52 / \mathrm{M}$ & 8 & Equivalent & 24 & 600 & L 3 vs 4 & $-10.5,-15.5,-6$ & $29 \%$ & 24 \\
\hline \multirow[t]{2}{*}{8} & $56 / \mathrm{M}$ & 4 & Tremor-dominant & $\begin{array}{l}\text { Not } \\
\text { available }\end{array}$ & 0 & L 2 vs 3 & $-10,-14.5,-7.5$ & $29 \%$ & 27 \\
\hline & & & & & & $\mathrm{R} 1$ vs 2 & $12,-15,-7.5$ & $25 \%$ & 25 \\
\hline 9 & $50 / \mathrm{M}$ & 2 & Equivalent & 30 & 960 & R 2 vs 3 & $12,-15,-7$ & $25 \%$ & 29 \\
\hline \multirow[t]{2}{*}{$11^{\mathrm{b}}$} & $61 / \mathrm{M}$ & 9 & Equivalent & 36 & 1,270 & L 2 vs 5 & $-11,-14.5,-8$ & $29 \%$ & 14 \\
\hline & & & & & & $\mathrm{R} 1$ vs 2 & $12.5,-12.5,-9$ & $21 \%$ & 15 \\
\hline $12^{\mathrm{b}}$ & 64/M & 25 & Hypokinetic-rigid & 46 & 1,410 & $\mathrm{~L} 2 \mathrm{~A}$ vs $3 \mathrm{~A}$ & $-11,-16,-7$ & $17 \%$ & 19 \\
\hline \multirow[t]{2}{*}{$13^{\mathrm{b}}$} & $52 / \mathrm{M}$ & 5 & Hypokinetic-rigid & 33 & $\begin{array}{l}850+ \\
\text { safinamide } \\
50 \mathrm{mg}^{\mathrm{c}}\end{array}$ & $\mathrm{L} 2 \mathrm{~B}$ vs $3 \mathrm{~B}$ & $-11.5,-16.5,-7$ & $12 \%$ & 27 \\
\hline & & & & & & R $2 \mathrm{C}$ vs 4 & $13.5,-16,-6$ & $8 \%$ & 25 \\
\hline \multirow[t]{2}{*}{$14^{\mathrm{b}}$} & $63 / \mathrm{F}$ & 8 & Hypokinetic-rigid & 46 & 1,110 & $\mathrm{~L} 2 \mathrm{C}$ vs $3 \mathrm{C}$ & $-9,-16.5,-9$ & $21 \%$ & 35 \\
\hline & & & & & & R 1 vs $2 A$ & $12,-14.5,-9$ & $21 \%$ & 32 \\
\hline \multirow[t]{2}{*}{15} & $57 / \mathrm{M}$ & 7 & Hypokinetic-rigid & 44 & 890 & $\mathrm{~L} 3 \mathrm{~B}$ vs $3 \mathrm{C}$ & $-11,-13,-8$ & $21 \%$ & 22 \\
\hline & & & & & & R $3 A$ vs 4 & $13.5,-13,-6.5$ & $21 \%$ & 21 \\
\hline \multirow[t]{2}{*}{16} & $60 / F$ & 12 & Hypokinetic-rigid & 22 & 1,360 & $\mathrm{~L} 1$ vs $2 \mathrm{~A}$ & $-9,-17.5,-9.5$ & $8 \%$ & 20 \\
\hline & & & & & & $\mathrm{R} 2 \mathrm{C}$ vs $3 \mathrm{C}$ & $11.5,-15.5,-9.5$ & $8 \%$ & 21 \\
\hline 17 & $64 / \mathrm{F}$ & 7 & Hypokinetic-rigid & 16 & 1,090 & $\mathrm{R} 2 \mathrm{C}$ vs $3 \mathrm{C}$ & $13.5,-14.5,-5$ & $12 \%$ & 15 \\
\hline \multicolumn{10}{|c|}{$\begin{array}{l}{ }^{\text {aSTN }} \text { probability map entries for selected contacts. The atlas maximum is } 0.46 \text {. } \\
\text { bPatient with freezing of gait according to score }>1 \text { at item } 3 \text { of the FOG-Q. } \\
\text { 'Safinamide } 50 \mathrm{mg} \text { is not included in the L-dopa equivalent dose as it is currently } \\
\text { colleagues. }{ }^{47}\end{array}$} \\
\hline
\end{tabular}

\section{Data Acquisition and Analysis}

LFPs from the STN were recorded with a portable electroencephalographic (EEG) amplifier at a sampling rate of $2,048 \mathrm{~Hz}$ (Porti amplifier, TMSi, Enschede, the Netherlands) using the open source software packages OpenBCI and Svarog. ${ }^{18}$ The portable amplifier was attached with a waist belt and a custommade pocket. The externalized leads were fixed with tape on the back of the neck. In addition, EEG from a few cortical Ag/ $\mathrm{AgCl}$ electrodes, positioned over sensorimotor and parietal areas, and electromyogram of bilateral leg muscles (tibialis anterior, biceps femoris, and rectus femoris) were assessed simultaneously. The water-based ground electrode was integrated in a wristband, and LFP and EEG data were recorded with an average reference. LFP and EEG data were rereferenced offline with a bipolar montage consisting of adjacent contacts in the vertical plane. This yielded 7 and 9 bipolar channels per STN depending on the electrode model, respectively. Data were obtained from 26 STNs. Two STNs were excluded due to a malfunctional extension or connector. Two further STNs had to be excluded because of severe movement 
A

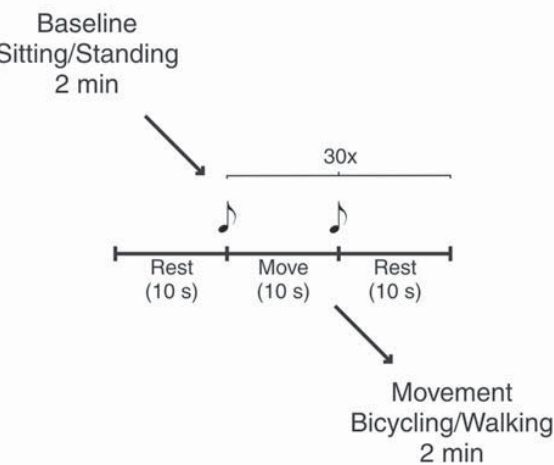

B

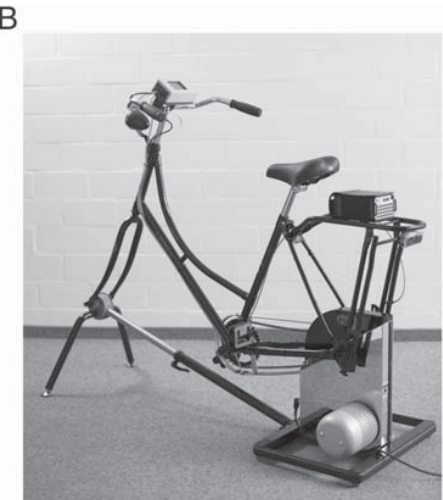

FIGURE 1: Experimental setup. (A) Schematic illustration of the experimental paradigm adapted from Storzer et al. ${ }^{14}$ Patients started with a 2-minute baseline rest period, that is, sitting on the bicycle or standing. This was followed by repeatedly alternating 10 seconds of movement and 10 seconds of rest with a further 10 seconds of rest at the beginning. This way, 30 instances of acoustically cued movement initiation and termination were captured. Subsequently, patients walked or pedaled continuously for 2 minutes. (B) The bicycle simulator consisted of a Dutch-style bicycle frame mounted on an ergometer. Pedal position was recorded simultaneously with all electrophysiological signals.

artifact contamination affecting all channels. Consequently, 22 STNs were included in the analysis.

Data were analyzed with the MATLAB-based FieldTrip toolbox ${ }^{19}$ using MATLAB R2015b (MathWorks, Natick, MA). Analysis was focused on LFPs. First, data were bandpass filtered offline $(1-1,000 \mathrm{~Hz})$. Next, data were visually inspected for movement artifacts and respective segments were excluded from further analysis.

SPECTRAL ANALYSIS. Power spectra were calculated for the baseline rest condition, that is, sitting or standing for bicycling or walking, respectively. Data were divided into 1-second segments with an overlap of $50 \%$, and the fast Fourier transform was calculated for each segment using a single Hanning taper. Afterward, spectra were averaged over segments. Event-related modulations of LFP power were analyzed by performing timefrequency decomposition locked to movement initiation and termination with a sliding Hanning window of 1-second length and a step size of 0.05 seconds. Power changes were expressed as the relative power change in decibels to the baseline rest condition. The goniometer signal was used to define movement initiation and termination in line with Storzer et al. ${ }^{14}$ Based on visual inspection, $3.5 \%$ of the selected initiations and $5.4 \%$ of the selected terminations had to be adapted.

CHANNEL SELECTION. We selected 1 bipolar channel per STN for the analysis. Based on Litvak and colleagues, ${ }^{20}$ the channel with the strongest change of spectral power in the 13to $35 \mathrm{~Hz}$ range (pooled over conditions) was selected. We verified that the corresponding channels also showed a baseline beta peak. The selected channel was the same for all movement conditions, to prevent channel selection from affecting the comparison between bicycling and walking. Due to nonfunctional contacts in Patients \#13 and \#15, the contact pair 2C-4 (Patient \#13) and 3B-3C (Patient \#15) had to be selected. Two bipolar channels had to be adapted due to movement artifact contamination in 2 patients ( $\# 5$ and $\# 17$ ). In these cases, the next possible channel with strong beta suppression was selected instead.

The Table lists the selected channels for each STN together with the Montreal Neurological Institute coordinates and the probability of being within the STN. Coordinates and the respective probabilities were determined as described by Hirschmann and colleagues ${ }^{21}$ and based on the probability maps of the STN derived from ultra-high-resolution $7 \mathrm{~T}$ magnetic resonance images provided by Forstmann et al. ${ }^{22}$ Electrode localization was not possible for 1 patient due to movement artifacts contaminating the computed tomography.

\section{Statistics}

To assess beta power changes before and after movement initiation, baseline-corrected power was averaged in the time window from -0.5 to 1 second. Likewise, power was averaged in the window from 0.5 to 2 seconds around movement termination. We verified that similar results were obtained when choosing different window sizes.

Differences in baseline-corrected power, cadence, and baseline log power were evaluated by fitting linear mixed-effects models using the package lme4 1.1-13 for $\mathrm{R}$ version 3.4.0. ${ }^{23} \mathrm{In}$ contrast to a conventional analysis of variance, linear mixedeffects models account for similarity between samples, for example, between values obtained from left and right STN of the same patient.

In each model, the fixed effects structure was defined as the 2-level factor movement type (bicycling or walking) and the 2-level factor patient group (freezer or nonfreezer). Additionally, the 2-level factor frequency band (low or high beta) was included in case the dependent variable was power. The definition of low $(13-22 \mathrm{~Hz})$ and high beta $(23-35 \mathrm{~Hz})$ was based on previous studies. ${ }^{12,13,24}$ All interactions were included in the model. Furthermore, we included the random factors patient and STN. STN was nested in patient.

Factors were sum coded. Normality and homoscedasticity of residuals were verified by visual inspection. Probability values 

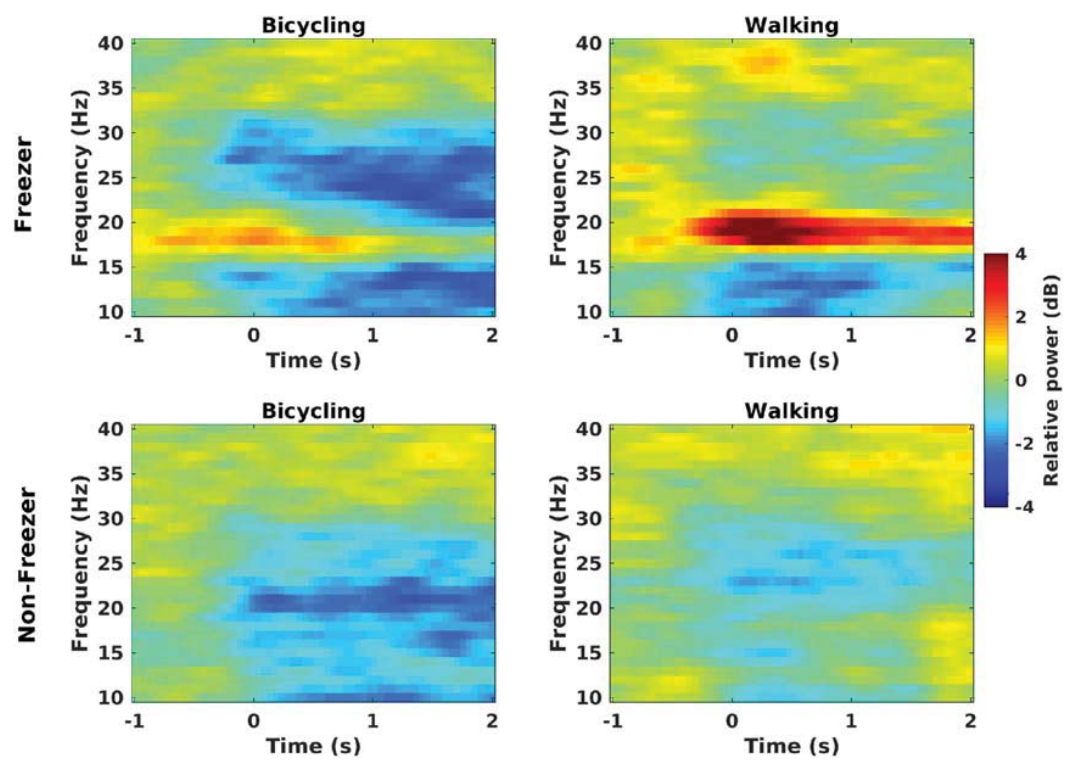

FIGURE 2: Time-frequency representation of movement initiation in freezers and nonfreezers. Grand average time-frequency plots showing power changes locked to movement initiation $(t=0)$ in freezers ( 9 subthalamic nuclei [STNs]) and nonfreezers (13 STNs) for bicycling and walking. Please note that grand average plots are plain averages across all STNs, not accounting for possible correlations within patients. Upper row: In freezers, bicycling is accompanied by a broadband beta power decrease and, briefly, by a slight power increase (red) in a narrow band around $18 \mathrm{~Hz}$, following movement initiation. A distinctive and sustained power increase in this band can also been seen in walking. Lower row: Beta power decreases (blue) are seen in both conditions in nonfreezers, but with a stronger beta power decrease in bicycling.

were obtained using Wald chi-square tests, as implemented in the Anova function of the car package $2.1-4 .{ }^{25}$ In case of interactions, post hoc tests were computed using the $\mathrm{R}$ package lsmeans $2.26-3,{ }^{26}$ and corrected for multiple testing using the Tukey correction. The $95 \%$ confidence interval (CI) is reported.

\section{Results}

\section{Cadence}

Cadence was significantly affected by movement type $\left(\beta=-3.89,95 \% \mathrm{CI}=-5.65\right.$ to $-2.13, \chi^{2}=13.95$, $p<0.01$ ), reflecting a higher cadence in walking than in bicycling. Cadence was on average $41.21 \mathrm{rpm} \quad(95 \%$ $\mathrm{CI}=35.93-46.50)$ for bicycling and $43.83 \mathrm{spm}(95 \%$ $\mathrm{CI}=38.55-49.11)$ for walking in nonfreezers and $39.33 \mathrm{rpm} \quad(95 \% \quad \mathrm{CI}=32.65-46.01)$ and $52.26 \mathrm{spm}$ $(95 \% \mathrm{CI}=45.56-58.94)$ in freezers. Moreover, the 2way interaction of group and movement was significant $\left(\beta=2.58,95 \%\right.$ CI $\left.=0.82-4.34, \chi^{2}=8.11, p<0.01\right)$. Post hoc tests confirmed that the consistent difference between bicycling and walking was more pronounced in patients with FOG $\left(t \operatorname{ratio}_{[11]}=-4.55, p<0.01\right)$.

\section{Beta Power Changes Locked to Movement Initiation}

Relative power changes locked to movement initiation were evident in the beta band both in bicycling and in walking for patients with and without FOG (Fig 2).
Relative beta power was on average $-0.82 \mathrm{~dB} \quad(95 \%$ $\mathrm{CI}=-1.65$ to -0.00$)$ for bicycling and $-0.59 \mathrm{~dB}(95 \%$ $\mathrm{CI}=-1.41$ to 0.24 ) for walking in nonfreezers and $-0.58 \mathrm{~dB}(95 \% \mathrm{CI}=-1.61$ to 0.44$)$ and $0.30 \mathrm{~dB}(95 \%$ $\mathrm{CI}=-0.72$ to 1.33$)$ in freezers. The mixed model revealed a significant effect of movement type $\left(\beta=-0.28, \quad \mathrm{CI}=-0.49 \quad\right.$ to $\quad-0.08, \quad \chi^{2}=5.60$, $p=0.02)$, reflecting a stronger beta power suppression in bicycling than in walking irrespective of group or frequency band (Fig 3A). Furthermore, the 2-way interaction of group and frequency was significant $(\beta=-0.32$, $95 \% \mathrm{CI}=-0.53$ to $-0.12, \chi^{2}=8.99, p<0.01$; see Fig $3 \mathrm{~B})$. Post hoc tests revealed a significant difference between low and high beta power in freezers $\left(t_{[60]}=2.65, p<0.05\right)$. Whereas nonfreezers displayed a power reduction in the whole beta band during bicycling and walking, movement was accompanied by a selective low beta power increase in freezers.

As depicted in Figure 2 (upper panel), the power increase observed in freezers was due to enhancement of a specific narrowband oscillation occurring at approximately $18 \mathrm{~Hz}$. It was particularly strong at movement onset - a time at which the remaining beta band was generally suppressed (Fig 4). Uncoupling of the $18 \mathrm{~Hz}$ oscillation from the remaining beta band was more pronounced during walking than during bicycling $(\beta=0.13$, 95\% CI $\left.=0.04-0.22, \chi^{2}=8.62, p<0.01\right)$. 

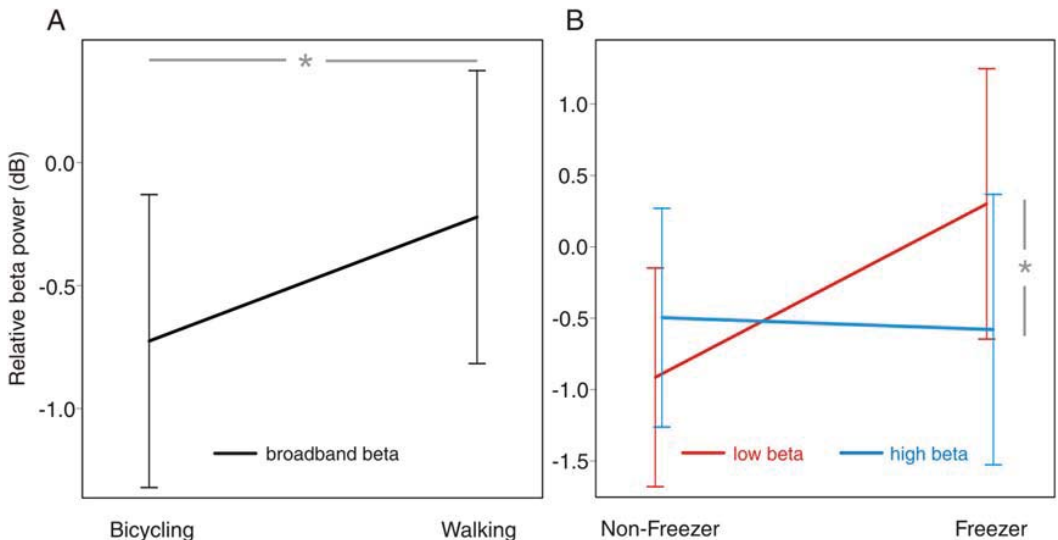

FIGURE 3: Predictor estimates and $95 \%$ confidence intervals for the mixed model on relative beta power around movement initiation. (A) Bicycling is accompanied by stronger beta power suppression relative to baseline than walking irrespective of group and frequency band. (B) Superimposed on the general effect of movement type on beta power, the interaction of group and frequency band was significant. Nonfreezers show a power reduction in the low and high beta band relative to baseline, irrespective of the movement type. In contrast, freezers display a selective power increase in the low but not in the high beta band, resulting in a significant difference between low and high beta power as indicated by the asterisk.

\section{Beta Power Changes Locked to Movement Termination}

Time-frequency representations of LFP power locked to movement termination showed a beta power increase relative to the baseline rest condition (beta rebound) for both bicycling and walking in patients with and without FOG (Fig 5). Rebound strength was affected by frequency band $(\beta=-0.45,95 \% \mathrm{CI}=-0.63$ to -0.28 , $\left.\chi^{2}=23.67, p<0.01\right)$, as it predominantly involved the high beta band. The rebound was not different between
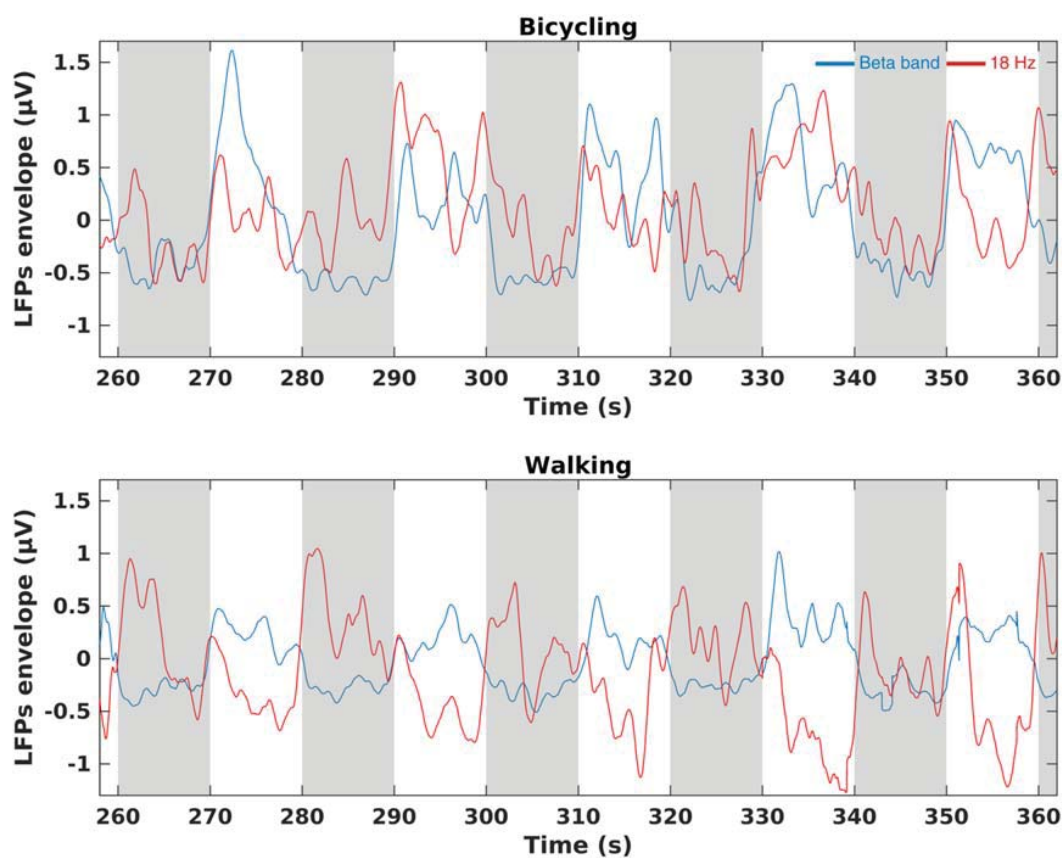

FIGURE 4: Modulation of the $\sim 18 \mathrm{~Hz}$ oscillation and the remaining beta band in freezers. Grand average envelopes of the $18 \mathrm{~Hz}$ oscillation (red) and the remaining beta band (blue) for patients with freezing of gait (FOG) are shown. Local field potential (LFP) data of each subthalamic nucleus (STN) were bandpass filtered around the frequency $( \pm 2 \mathrm{~Hz})$ showing the strongest movement-related power increase. Next, envelopes of the filtered data were computed by taking the Hilbert transform. Envelopes were filtered by a moving average filter of length 2,500 samples and correlated with the envelope of the remaining beta band. Please note that grand average plots are plain averages across all STNs, not accounting for possible correlations within patients. The gray boxes represent 5 of the 30 periods of movement, that is, bicycling and walking. Narrowband and broadband beta envelopes are uncoupled during walking but not during cycling in patients with FOG. Modulation of the $\sim 18 \mathrm{~Hz}$ oscillation is increased at the start and end of the movement phases. 

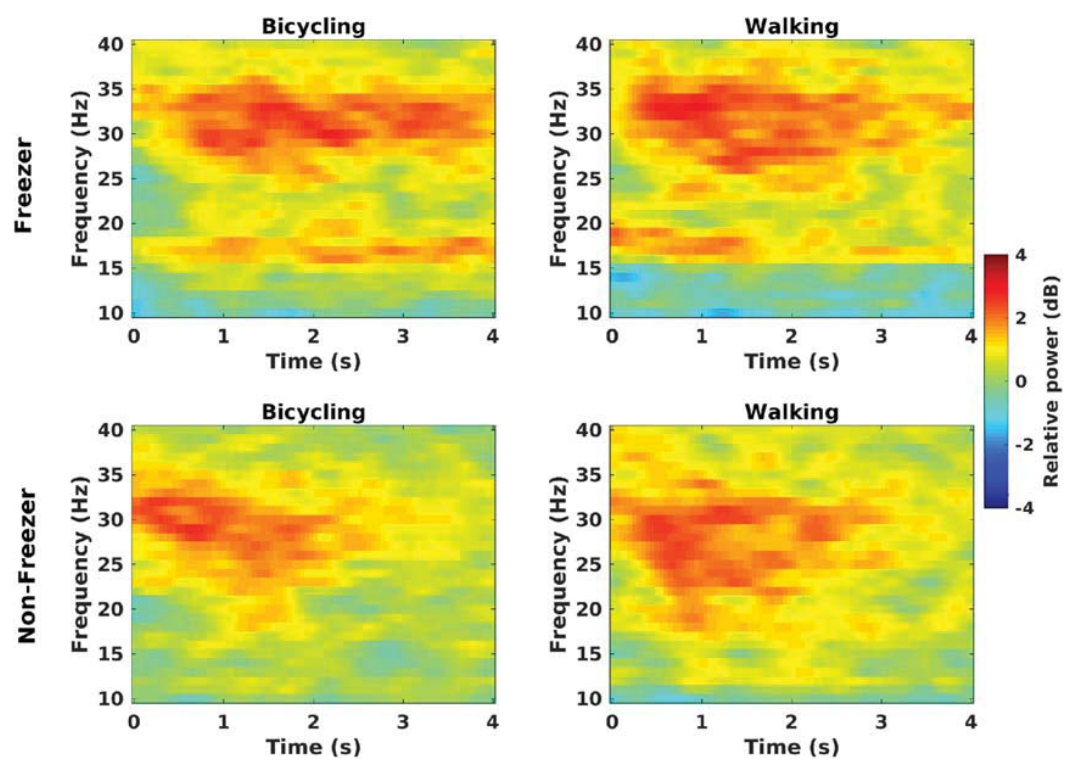

FIGURE 5: Time-frequency representation of movement termination in freezers and nonfreezers. Grand average timefrequency plots show power changes locked to movement termination $(t=0)$ in freezers ( 9 subthalamic nuclei [STNs]) and nonfreezers ( 13 STNs) for bicycling and walking. Please note that grand average plots are plain averages across all STNs, not accounting for possible correlations within patients. High beta power rebound $(23-35 \mathrm{~Hz})$ upon movement termination is observable in both conditions and groups and is of similar magnitude.

movement conditions $(\beta=-0.06,95 \% \mathrm{CI}=-0.24$ to $\left.0.11, \chi^{2}=0.72, p=0.40\right)$ or patient groups $(\beta=0.02$, $95 \% \mathrm{CI}=-0.37$ to $\left.0.41, \chi^{2}=0.01, p=0.93\right)$.

\section{Differences in Baseline Beta Power}

To test the importance of movement for the observed modulations, we repeated the analysis for beta power at baseline (sitting or standing). The interaction between patient group and frequency band reached significance $(\beta=-0.46,95 \%$ $\mathrm{CI}=-0.91$ to $\left.-0.02, \chi^{2}=3.87, p<0.05\right)$. Post hoc tests were carried out between freezers and nonfreezers, but only showed a trend for increased low beta power in freezers $(t$ ratio $_{[10.09]}=-2.16, \quad p=0.06$; high-beta: $t$ ratio $_{[10.09]}=$ $-1.40, p=0.19)$. Whether patients were standing or sitting did not affect baseline power $(\beta=0.21,95 \%$ $\mathrm{CI}=-0.23$ to $\left.0.66, \chi^{2}=0.71, p=0.40\right)$.

\section{Discussion}

We demonstrate for the first time that beta band activity in the parkinsonian STN is modulated differently by bicycling and walking. We found greater beta power suppression in bicycling relative to walking, whereas the beta rebound upon movement termination was equally strong. Importantly, patients with FOG exhibited a low beta power increase during movement — a characteristic oscillation around $18 \mathrm{~Hz}$. This apparently abnormal oscillation may comprise a signature of altered activity in the motor system and susceptibility to freezing that has not been described before.

\section{Stronger Beta Power Suppression in Bicycling}

We found bicycling to be associated with stronger suppression of beta oscillations relative to walking, in line with previous observations in the sensorimotor cortex of healthy participants. ${ }^{14}$ Beta rebound after movement termination, however, was of similar magnitude. Thus, the difference in beta power modulation between bicycling and walking was specific to beta power suppression during movement. It is not unlikely that this is a crucial part of the physiological mechanism explaining why bicycling ability is preserved in Parkinson disease and more importantly, why bicycling has a therapeutic effect. ${ }^{27}$

Some functional differences in the cerebral networks underlying bicycling and walking would be expected, as they differ in some aspects such as sensorimotor input, postural control, and coordination. It remains to be clarified which of these differences are associated with the stronger beta power suppression. Postural control during standing is impaired in Parkinson disease patients, with impairment and decline being more pronounced in freezers. ${ }^{28,29}$ However, after comparing beta baseline power between the different movement conditions, that is, sitting and standing, no difference was found. Consequently, stronger beta power suppression during bicycling is not likely to be due to reduced need for postural control.

A key difference could be the more continuous nature of bicycling, which is associated with persistent tactile feedback to both feet and lacks the stationary 
phases that are part of walking. Thus, the likelihood of the subsequent movement is higher in bicycling, which has been related to beta power suppression. ${ }^{30}$ Furthermore, leg movements in bicycling have fewer degrees of freedom due to the fixed pedal trajectory, regardless of the exact foot placement. In consequence, the limb movements required for bicycling are less complex than for walking and could impose a lower computational load on the motor system. In line with this interpretation, a previous study of our group found stronger gait phase-dependent power modulations in walking compared to bicycling in the sensorimotor cortex of healthy participants, ${ }^{14}$ which could be indicative of a reduced demand for cortical monitoring of ongoing movement.

\section{Low Beta Power Increase in Patients with FOG}

In line with a previous report by Singh and colleagues, ${ }^{13}$ freezers showed increased low beta power during movement. More specifically, patients with FOG displayed an abnormal $\sim 18 \mathrm{~Hz}$ oscillation in the STN that was not present in Parkinson disease patients without FOG. From where might this oscillation originate and what are its implications?

FUNCTIONAL ROLE. Due to its predominance in the OFF medication state and its corresponding reduction by both medication and DBS, ${ }^{24,31}$ the lower beta range is assumed to be particularly associated with antikinetic symptoms. Thus, the $\sim 18 \mathrm{~Hz}$ oscillation might reflect a movement-inhibiting signal throughout the motor network.

At the descriptive level, this altered oscillatory activity in freezers was intensified during walking. However, a specific difference between bicycling and walking in freezers could not be confirmed statistically. Although this might be due to small subgroup sample sizes, the current data point to a small effect, if any, which might be difficult to detect even with larger samples. Alternatively, the $18 \mathrm{~Hz}$ oscillation might be a general signature of altered basal ganglia activity that distinguishes freezers from nonfreezers irrespective of the motor state. The interaction between frequency band and patient group regarding beta baseline power supports this interpretation, as it indicates a difference between patient groups even at rest.

As the low beta power increase was seen irrespective of actual freezing episodes, it likely reflects a predisposition to freeze. It might be boosted by interference in the corticobasal ganglia loops due to additional response conflict or motor, cognitive, or limbic load, thereby triggering a freezing episode. ${ }^{10,11}$
ANATOMICAL ORIGIN. Interestingly, phase synchronization in the beta band over the left prefrontal area was found to be associated with increased susceptibility to upper limb freezing. ${ }^{32}$ The STN receives direct cortical projections from frontal areas via the hyperdirect pathway, ${ }^{33}$ which is assumed to mediate a top-down stopping signal. ${ }^{34}$ Thus, an excessive cortically driven inhibitory signal might manifest as an abnormal $\sim 18 \mathrm{~Hz}$ oscillation in the STN of freezing patients. Alternatively, the $\sim 18 \mathrm{~Hz}$ oscillation might emerge from the interaction between STN and $\mathrm{GPe}^{35}$ or originate in the striatum due to increased levels of cholinergic drive as a consequence of dopamine depletion. ${ }^{36}$ Results of a recent study analyzing functional interactions between the STN, motor cortex, and pedunculopontine nucleus during walking in hemiparkinsonian rats suggest the STN proper as a potential source of increased beta oscillations at around $18 \mathrm{~Hz}^{37}$

POWER INCREASE OR FREQUENCY SHIFT? Changes in baseline-corrected power, such as the $18 \mathrm{~Hz}$ power increase reported here, can sometimes be a consequence of a movement-related shift in peak frequency. ${ }^{38,39}$ Although we observed a clear shift in 1 of the 5 patients with FOG in non-baseline-corrected power (data not shown), a specific power increase at $18 \mathrm{~Hz}$ without a clear shift was observed in the remaining patients. Due to the limited sample size, we cannot definitively say which of the 2 patterns may best represent freezers in general. Further research is needed to clarify this issue.

WHY ARE FREEZERS ABLE TO BICYCLE? Our present results add substantially to the clarification of this question by providing electrophysiological correlates of the risk of freezing and demonstrating differences between bicycling and walking. We found that bicycling suppresses broadband beta power. Hence, the effect of bicycling is reminiscent of the therapeutic effects of DBS and medication. ${ }^{31,40-42}$ We explain the comparably low level of beta power during bicycling by a comparably low computational load on the motor system. This low load may decrease the risk of interference between the parallel but separated corticobasal ganglia loops, and thus the risk of freezing. ${ }^{10,11}$ If the movement sequence is made more complex, for example, by uncoupling the pedals of the bike, the load is increased and patients with FOG show irregular pedaling patterns with the return of intermittent cessations. ${ }^{43}$ Likewise, freezing was found to be partially alleviated by using a "walk-bicycle," that is, a bicycle without pedals and with a low seat. ${ }^{44}$ Locomotion with the walk-bicycle still requires alternating stepping movements of the legs but reduces the need for postural control and lateral weight shifts that were shown to be 
abnormally coupled in patients with FOG. ${ }^{45}$ In summary, we suggest that bicycling ability remains unaffected in most patients because it has a lower computational load on motor networks. ${ }^{7,8}$

\section{Methodological Considerations}

A methodological challenge in LFP studies on different types of movements lies in movement-related artifacts. We carefully inspected our data and excluded heavily contaminated data. Nevertheless, movement artifacts remained in our data to some extent, particularly in the walking condition. However, these artifacts were found to be restricted to lower frequencies $<10 \mathrm{~Hz}$, and thus, could not influence the presented results in the beta frequency band.

The limited availability of suitable patients restricted subgroup sample sizes, lowering statistical power, and the investigated cohort represents Parkinson disease patients selected for DBS therapy rather than the general patient population. Nevertheless, our data are in line with previous research ${ }^{13}$ and provide a promising basis for future studies. Such work should characterize the $18 \mathrm{~Hz}$ oscillation further, for example, by investigating possible modulations during actual freezing episodes, and should include a representative number of patients with and without FOG, allowing for statistical testing also with regard to different types of FOG. Finally, patients were identified as freezers according to the FOG-Q. Future studies should include an objective examination of FOG and include only patients with definite acute FOG. ${ }^{46}$

\section{Conclusion}

Our study provides important first evidence for differences between bicycling and walking in the oscillatory activity of the STN in Parkinson disease patients. Our results indicate that bicycling is accompanied by a stronger movement-related suppression of pathological beta oscillations compared to walking, with an influence on STN beta rhythms reminiscent of the effects of DBS. Furthermore, freezers exhibited a selective, prolonged, distinct, and narrowband beta oscillation locked to the initiation of movement at $\sim 18 \mathrm{~Hz}$. This oscillation was seen irrespective of actual freezing episodes, and was descriptively weaker and rather brief in duration upon initiation of bicycling, signifying that it likely reflects susceptibility to freezing. Bicycling facilitates suppression of overall beta power, and we speculate that, due to its continuous nature, it is computationally less demanding than walking and thus less prone to overloading the networks involved in locomotion.

\section{Acknowledgment}

This work was supported by a grant from the Jacques and Gloria Gossweiler Foundation. Further support was received from the Zukunftskolleg of the University of Konstanz (S.S.D.), Heinrich Heine University Strategic Research Funds (M.B.), and the research commission of the medical faculty of Heinrich Heine University (9772562, M.B.). J.H. is supported by the German Academic Exchange Service (P.R.I.M.E. 2015). L.S. was supported by travel grants from the Boehringer Ingelheim Foundation and the German Academic Exchange Service.

We thank the patients who participated in this study; N. Sharma for assistance with constructing and programming the bicycle simulator; the neurologists and neurosurgeons of the University Hospital Düsseldorf for their support; and Drs A. Klepp and H. Schwender for their assistance with implementing linear mixed-effects models.

\section{Author Contributions}

L.S., M.B., J.H., A.S., D.S., and S.S.D. contributed to the design of the experiment and M.G., D.S., S.S.D., L.S., and M.B. to the experimental setup. L.S., M.B., J.H., O.A., A.S., and S.S.D. were involved in data analysis and interpretation and L.S., J.H., J.V., A.S., and M.B. in data acquisition. L.S., M.B., J.H., A.S., and S.S.D. wrote the article.

\section{Potential Conflicts of Interest}

J.V. receives consultant fees and A.S. consultant and lecture fees from Boston Scientific, Medtronic, and St. Jude Medical, which manufacture the DBS systems used in this study.

\section{References}

1. Peterson DS, Horak FB. Neural control of walking in people with parkinsonism. Physiology 2016;31:95-107.

2. Oswal A, Brown P, Litvak V. Synchronized neural oscillations and the pathophysiology of Parkinson's disease. Curr Opin Neurol 2013;26:662-670.

3. Engel AK, Fries P. Beta-band oscillations-signalling the status quo? Curr Opin Neurobiol 2010;20:156-165.

4. Nutt JG, Bloem BR, Giladi N, et al. Freezing of gait: moving forward on a mysterious clinical phenomenon. Lancet Neurol 2011; 10:734-744

5. Chee R, Murphy A, Danoudis M, et al. Gait freezing in Parkinson's disease and the stride length sequence effect interaction. Brain 2009;132:2151-2160.

6. Nieuwboer A, Dom R, De Weerdt W, et al. Abnormalities of the spatiotemporal characteristics of gait at the onset of freezing in Parkinson's disease. Mov Disord 2001;16:1066-1075.

7. Snijders AH, van Kesteren M, Bloem BR. Cycling is less affected than walking in freezers of gait. J Neurol Neurosurg Psychiatry 2012;83:575-576. 
8. Snijders AH, Toni I, Ružička E, Bloem BR. Bicycling breaks the ice for freezers of gait. Mov Disord 2011;26:367-371.

9. Raasch CC, Zajac FE. Locomotor strategy for pedaling: muscle groups and biomechanical functions. J Neurophysiol 1999;82:515525.

10. Lewis SJG, Barker RA. A pathophysiological model of freezing of gait in Parkinson's disease. Parkinsonism Relat Disord 2009;15: 333-338.

11. Lewis SJG, Shine JM. The next step: a common neural mechanism for freezing of gait. Neuroscientist 2016;22:72-82.

12. Toledo JB, López-Azcárate J, Garcia-Garcia D, et al. High beta activity in the subthalamic nucleus and freezing of gait in Parkinson's disease. Neurobiol Dis 2014;64:60-65.

13. Singh A, Plate A, Kammermeier S, et al. Freezing of gait-related oscillatory activity in the human subthalamic nucleus. Basal Ganglia 2013;3:25-32.

14. Storzer $\mathrm{L}$, Butz $\mathrm{M}$, Hirschmann J, et al. Bicycling and walking are associated with different cortical oscillatory dynamics. Front Hum Neurosci 2016;10:61

15. Giladi N, Shabtai $H$, Simon ES, et al. Construction of freezing of gait questionnaire for patients with parkinsonism. Parkinsonism Relat Disord 2000;6:165-170.

16. Özkurt TE, Butz M, Homburger $M$, et al. High frequency oscillations in the subthalamic nucleus: a neurophysiological marker of the motor state in Parkinson's disease. Exp Neurol 2011;229:324331

17. Gratkowski M, Storzer L, Butz M, et al. BrainCycles: experimental setup for the combined measurement of cortical and subcortical activity in Parkinson's disease patients during cycling. Front Hum Neurosci 2017;10:685.

18. Durka PJ, Kuś R, Żygierewicz J, et al. User-centered design of brain-computer interfaces: OpenBCl.pl and $\mathrm{BCl}$ Appliance. Bull Polish Acad Sci Tech Sci 2012;60:427-431.

19. Oostenveld R, Fries P, Maris E, Schoffelen J-M. FieldTrip: Open Source Software for Advanced Analysis of MEG, EEG, and Invasive Electrophysiological Data. Comput. Intell. Neurosci. 2011; 2011:1-9.

20. Litvak V, Eusebio A, Jha A, et al. Movement-related changes in local and long-range synchronization in Parkinson's disease revealed by simultaneous magnetoencephalography and intracranial recordings. J Neurosci 2012;32:10541-10553.

21. Hirschmann J, Butz M, Hartmann CJ, et al. Parkinsonian rest tremor is associated with modulations of subthalamic highfrequency oscillations. Mov Disord 2016;31:1551-1559.

22. Forstmann BU, Keuken MC, Jahfari S, et al. Cortico-subthalamic white matter tract strength predicts interindividual efficacy in stopping a motor response. Neuroimage 2012;60:370-375.

23. Bates $D$, Mächler M, Bolker BM, Walker SC. Fitting linear mixedeffects models using Ime4. J Stat Softw 2015;67:1-48.

24. López-Azcárate J, Tainta M, Rodríguez-Oroz MC, et al. Coupling between beta and high-frequency activity in the human subthalamic nucleus may be a pathophysiological mechanism in Parkinson's disease. J Neurosci 2010;30:6667-6677.

25. Fox J, Weisberg S. An $\{R\}$ companion to applied regression. 2nd ed. Thousand Oaks, CA: Sage, 2011.

26. Lenth RV. Least-squares means: the R package Ismeans. J Stat Softw 2016;69:1-33.

27. Alberts $\mathrm{JL}$, Linder $\mathrm{SM}$, Penko $\mathrm{AL}$, et al. It is not about the bike, it is about the pedaling. Exerc Sport Sci Rev 2011;39:177-186.

28. Vervoort G, Bengevoord A, Strouwen C, et al. Progression of postural control and gait deficits in Parkinson's disease and freezing of gait: a longitudinal study. Parkinsonism Relat Disord 2016:28: 73-79.

29. Schlenstedt C, Muthuraman M, Witt K, et al. Postural control and freezing of gait in Parkinson's disease. Parkinsonism Relat Disord 2016:24:107-112

30. Jenkinson $N$, Brown P. New insights into the relationship between dopamine, beta oscillations and motor function. Trends Neurosci 2011:34:611-618.

31. Oswal A, Beudel M, Zrinzo L, et al. Deep brain stimulation modulates synchrony within spatially and spectrally distinct resting state networks in Parkinson's disease. Brain 2016;139:1482-1496.

32. Scholten M, Govindan RB, Braun C, et al. Cortical correlates of susceptibility to upper limb freezing in Parkinson's disease. Clin Neurophysiol 2016;127:2386-2393.

33. Nambu A, Takada M, Inase M, Tokuno H. Dual somatotopical representations in the primate subthalamic nucleus: evidence for ordered but reversed body-map transformations from the primary motor cortex and the supplementary motor area. J Neurosci $1996 ; 16: 2671-2683$.

34. Aron AR, Herz DM, Brown $P$, et al. Frontosubthalamic circuits for control of action and cognition. J Neurosci 2016;36:11489-11495.

35. Holgado AJN, Terry JR, Bogacz R. Conditions for the generation of beta oscillations in the subthalamic nucleus-globus pallidus network. J Neurosci 2010:30:12340-12352.

36. McCarthy MM, Moore-Kochlacs C, Gu X et al. Striatal origin of the pathologic beta oscillations in Parkinson's disease. Proc Natl Acad Sci U S A 2011;108:11620-11625.

37. Li M, Zhou M, Wen $P$, et al. The network of causal interactions for beta oscillations in the pedunculopontine nucleus, primary motor cortex, and subthalamic nucleus of walking parkinsonian rats. Exp Neurol 2016;282:27-36.

38. Foffani G, Bianchi AM, Baselli G, Priori A. Movement-related frequency modulation of beta oscillatory activity in the human subthalamic nucleus. J Physiol 2005;568(pt 2):699-711.

39. Alegre $M$, Rodríguez-Oroz MCC, Valencia $M$, et al. Changes in subthalamic activity during movement observation in Parkinson's disease: is the mirror system mirrored in the basal ganglia? Clin Neurophysiol 2010;121:414-425.

40. Quinn EJ, Blumenfeld Z, Velisar A, et al. Beta oscillations in freely moving Parkinson's subjects are attenuated during deep brain stimulation. Mov Disord 2015;30:1750-1758.

41. Kühn AA, Kupsch A, Schneider G-H, Brown P. Reduction in subthalamic $8-35 \mathrm{~Hz}$ oscillatory activity correlates with clinical improvement in Parkinson's disease. Eur J Neurosci 2006;23: 1956-1960.

42. Kühn AA, Tsui A, Aziz T, et al. Pathological synchronisation in the subthalamic nucleus of patients with Parkinson's disease relates to both bradykinesia and rigidity. Exp Neurol 2009;215:380-387.

43. Abe $K$, Asai $Y$, Matsuo $Y$, et al. Classifying lower limb dynamics in Parkinson's disease. Brain Res Bull 2003;61:219-226.

44. Stummer C, Dibilio V, Overeem S, et al. The walk-bicycle: a new assistive device for Parkinson's patients with freezing of gait? Parkinsonism Relat Disord 2015;21:755-757.

45. Jacobs JV, Nutt JG, Carlson-Kuhta $P$, et al. Knee trembling during freezing of gait represents multiple anticipatory postural adjustments. Exp Neurol 2009;215:334-341.

46. Snijders $A H$, Haaxma $C A$, Hagen $Y J$, et al. Freezer or non-freezer: clinical assessment of freezing of gait. Parkinsonism Relat Disord 2012;18:149-154.

47. Tomlinson CL, Stowe R, Patel S, et al. Systematic review of levodopa dose equivalency reporting in Parkinson's disease. Mov Disord 2010;25:2649-2653. 